\title{
Batch-assembled multi-level micromachined mechanisms from bulk silicon
}

\author{
Yogesh Gianchandani and Khalil Najafi \\ Center for integrated Sensors and Circuits, University of Michigan, 1246 EECS \\ Building, 1301 Beal Avenue, Ann Arbor, MI 48109-2122, USA
}

Received 23 March 1992, accepted for publication 13 April 1992

\begin{abstract}
This paper reports on the development of a new technology intended for the wafer level fabrication and assembly of fully integrated micromechanisms. The technology is based on a boron=doped bulk silicon dissolved wafer process that has been used to fabricate a variety of micromechanical devices. The overall process utilizes three wafers: two silicon and one glass. All the major mechanical elements, including gears and micromotors, are fabricated from one silicon wafer, whereas the mechanical links between these elements are fabricated from a second silicon wafer. These wafers are successively aligned and bonded to a glass wafer which forms the substrate and are then dissolved in EDP to free the mechanisms. This procedure permits wafer-level batch assembly of

micromechanical systems. A number of bulk silicon electrostatic micromotors 5 $10 \mu \mathrm{m}$ thick and gear trains have been fabricated and linked to each other on the same chip. The results show that this is a very viable technology that allows mechanical power to be coupled between various devices.
\end{abstract}

\section{Introduction}

The development of complete micromechanical systems on a single chip has become an important goal for many integrated microsystems. These systems must incorporate microsensors, microactuators and electronics for fully independent operation. Extensive progress has been made in the development of solid state sensors and integrated electronics over the past few decades, and important steps have been taken in developing microactuators. Many devices, including electrostatic micromotors, joints and hinges, gear trains, and others have been fabricated and their basic operation has beeen demonstrated. However, the realization of fully integrated microsystems in which these mechanical devices can generate useful work under the control of solid state sensors and circuitry has yet to be accomplished. One of the significant challenges in moving towards this goal is the mechanical coupling of power between individual mechanical components of the system, especially where micromotors are involved.

A large variety of electrostatic micromotors have been fabricated in the past few years. Using surface micromachining, for example, researchers have made these devices from deposited thin films of materials such as polycrystalline silicon and tungsten, and operated them for extended periods of time [1]. There has been no demonstration, however, of mechanical work being transferred from an electrostatic micromoior to other elements in a system. One of the challenges in building mechanical linkages with surface micromachining is in planarizing the coupling beams, particularly if they are to be elevated above the level of the micromotors and gear trains. Some researchers have been successful in coupling magnetic micromotors to gear trains using fabrication methods based on x-ray lithography and metal electroforming (LIGA) [2]. Although this technology promises to have a major impact on the future of micromechanical systems, it requires sophisticated $\mathrm{x}$-ray equipment that is not widely available. Furthermore, the mechanisms that have been demonstrated constrain the links to lie in the plane of the devices and cannot be directly applied to electrostatic micromotors. This technology may also require some degree of individual component assembly, particularly for the important class of four-bar linkages.

In this paper we describe a bulk silicon fabrication technology that allows the batch fabrication and assembly of complete micromechanical systems. One of the key advantages of this technology is that mechanical components, including electrostatic micromotors, are easily coupled using elevated links that do not interfere with their operation. In a two-level system, with one level reserved for devices such as micromotors and gear trains, and the other level reserved for coupling links, it is possible to implement a variety of four-bar linkages, including slider-crank mechanisms and double-rocker mechanisms [3]. Mechanisms in which the output link is cranked can easily be implemented if the coupling link is 
extended past the output link, perhaps at an angle, to another motor driven synchronously with the first [4]. Using the proposed technology we have linked electrostatic micromotors to gear trains.

\section{Fabrication}

As the test vehicle for our fabrication technology we have chosen a simple mechanism based on electrostatic micromotors and gear trains mechanically linked together (figure 1). The mechanism was designed only to illustrate the capabilities of the proposed fabrication process and is not intended to fulfill any specific function. The micromotor has 12 stator poles and eight rotor poles, similar to those presented by others, and is driven by laterally applied electrostatic forces [1]. Mechanical energy is coupled by a link out of the micromotor into a gear resting nearby. The coupling link includes a beam

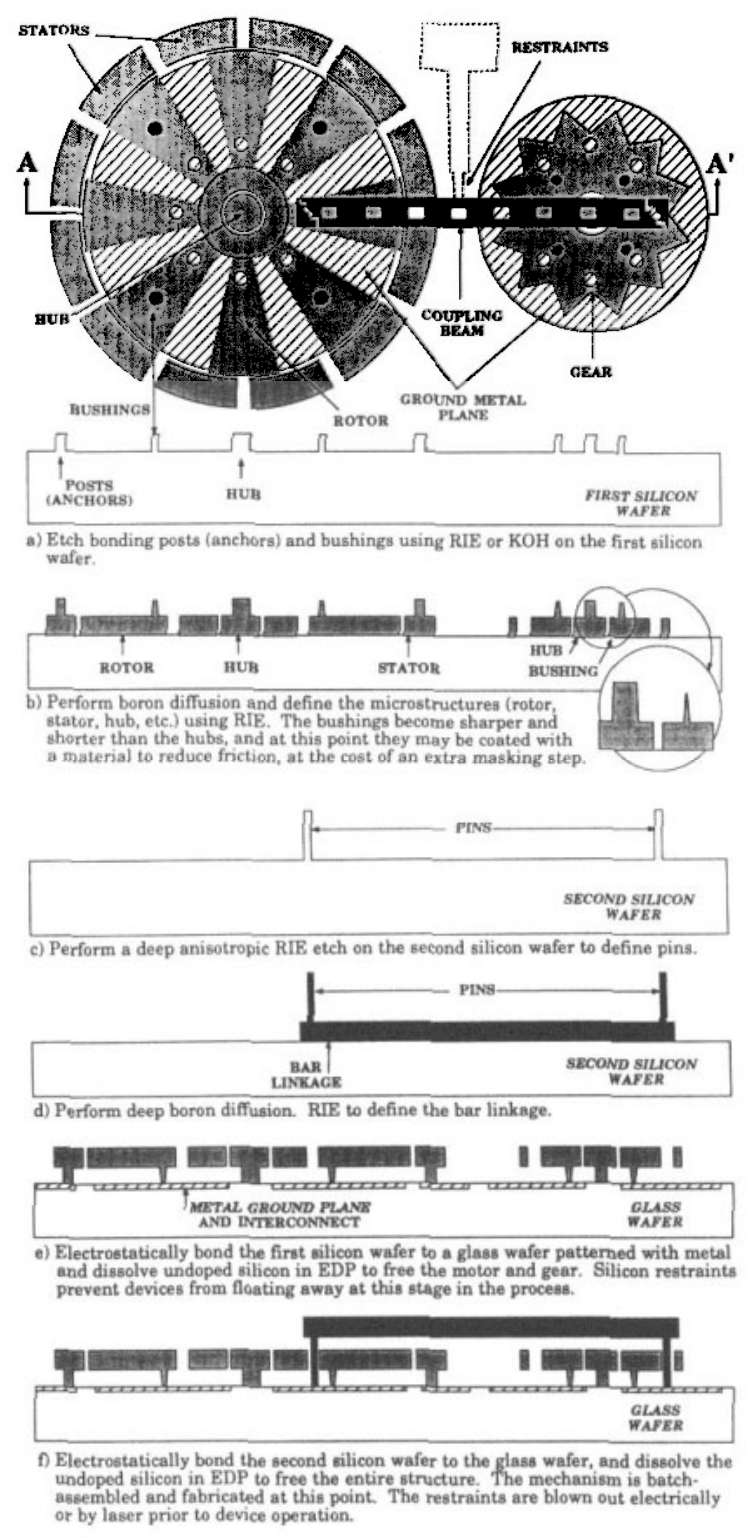

Figure 1. Process sequence for batch fabrication and assembly of micromotor-driven mechanisms. extended between two pins that penetrate through perforations in the rotor and the gear, resting on the underlying glass substrate.

The fabrication sequence requires the processing of two standard p-type (100) silicon wafers and a single glass wafer (figure 1). The silicon wafers require two masks each, and the glass wafer requires one. The first silicon wafer is patterned with photoresist and recessed by reactive ion etching (RIE) using a mixture of $\mathrm{SF}_{6}$ and $\mathrm{O}_{2}$ at a pressure of $7 \mathrm{mT}$ Torr and a power density of $85 \mathrm{~mW} \mathrm{~cm}^{-2}$. The etch rate, depending upon the loading, is about $800 \AA \mathrm{min}^{-1}$, and the trench side walls are $3-6^{\circ}$ off the vertical. All regions are etched except those that will eventually be bonded to the glass (e.g., anchors of the hubs and the stators of the motors), and the bushings that levitate the totors and the gears (figure 1(a)). An unmasked boron diffusion is performed next at $1175^{\circ} \mathrm{C}$. The diffusion depth determines the thickness of the rotor blades and can be in excess of $10 \mu \mathrm{m}$. The wafer is then patterned with an appropriate mask for RIE that traverses entirely through the boron diffusion, defining the rotor blades, gears, etc (figure $1(b)$ ). This etch must be capable of forming fine features and gaps with vertical sidewalls. We have developed a recipe for it based on a mixture of $\mathrm{SF}_{6}$ and $\mathrm{CCl}_{2} \mathrm{~F}_{2}$ gases [5]. The etch rate is $1400 \AA \min ^{-1}$, and the side walls are smooth and nearly vertical. This RIE also defines restraints that prevent the moving parts of the devices from floating away in subsequent ethylenediamine pyroacatechol (EDP) etches. The restraints are destroyed prior to device operation by current or laser pulses.

Note that the fabrication of the bushings is achieved at no cost. The bushings are made at the same time that the bonding posts are defined by the initial RIE step (figure $1(a)$ ). They are typically made into circular posts that have sufficiently small diameters so that, after the initial RIE and the boron diffusion step, their tops are somewhat rounded and slightly recessed below the initial surface of the wafer (figure 1(b)). An added advantage of this fabrication technique is that the top surface of the bushings can be coated (at the cost of an extra mask) with a material such as silicon nitride to minimize friction and/or to enhance resistance to wear.

The second silicon wafer is processed in essentially the same manner as the first, but with two different masks. The first of these defines, with deep anisotropic RIE, the pins that pass through perforations in the rotor blades of the micromotor (figure $1(c)$ ). The etched depth must exceed, by several microns, the sum of the depths of the initial recess and the boron diffusion performed on the first silicon wafer. This is recessary to ensure that the weight of the coupling link rests on the substrate and not on the elements on the first level. The second silicon wafer is then subjected to an unmasked boron diffusion to define the thickness of the coupling links. This diffusion must be deep enough to dope highly the entire body of the pins. Since the pins are $10 \mu \mathrm{m}$ in diameter, the diffusion depth and the thickness of the beams should be at least $5 \mu \mathrm{m}$. The diffusion should also be deep enough to ensure the rigidity of the beams. (Rigidity is also a design 
parameter for the width of the beams.) After this diffusion, the second mask is used to define the coupling links themselves by etching through the boron-diffused layer with RIE (figure $1(d)$ ). The material used to mask the coupling links against this etch must be able to provide effective coverage for the near-vertical side walls of the pins. If the side-wall coverage is poor, the pins defined by the previous etch will experience further undercutting in this step. Although we have had some limited success using metal masks for this RIE, we believe that thermally grown oxide may hold greater promise. Efforts are at present under way to resolve this issue.

The glass substrate is patterned with metal interconnect for the silicon devices. The metal is also used to form ground planes underneath the devices, which are helpful in two respects. First, during normal operation of the device they prevent a build-up of charge between the devices and the substrate, thereby minimizing the likelihood of clamping due to electrostatic attraction. Second, they can be used to prevent the bonding of certain portions of the devices, including the pins of the coupling links and the bushings, to the glass substrate. Electrical lead transfer to the stationary elements of a device (e.g. the stators of an electrostatic micromotor) is accomplished by partially extending the interconnect metal into the region to be bonded, causing the silicon and the metal to be tightly pressed together. In order to establish a good contact it is necessary to have the top surface of the metallized region protrude a little $(500 \AA)$ above the field surface of the glass. If this protrusion becomes too large, the metal will prevent the silicon and the glass from coming into intimate contact and prevent the formation of a good bond. At the same time, it is usually undesirable to limit the thickness of the interconnect metal to merely $500 \AA$. In order to overcome this conflict the glass wafer is recessed in the areas to be metallized.

The first step in processing the (Corning 7740) glass wafer is to pattern it with positive photoresist. The wafer is then immersed in $\mathrm{BHF}$ to recess the exposed regions by about $1500 \AA$ and then coated with $200 \AA$ of $\mathrm{Ti}$ and $1500 \AA$ of $\mathrm{Pt}$. Next, the photoresist is removed, causing the metal to be lifted off from the field regions [5]. The glass wafer is then grooved along the scribe lines in between the dice, subjected to a pre-bond organic clean, aligned and electrostatically bonded to the first silicon wafer. The bonding is performed at $400-450^{\circ} \mathrm{C}$, with $1000 \mathrm{~V}$ applied across the silicon-glass sandwich for $15-$ 30 min. As mentioned before, only those regions in which the silicon directly contacts the glass become bonded. The sandwich is then immersed in EDP to dissolve away the undoped silicon, leaving the heavily boron-doped motors and the gear trains resting on the glass substrate (figure 1(e)). The glass wafer is then subjected to another organic clean in preparation for bonding to the second silicon wafer. The second silicon wafer is aligned such that the pins of the links pass through perforations in the rotors and gears resting on the glass. As a high voltage is applied during the second bond, mechanical elements from the first wafer are deflected towards the glass because of electrostatic attraction. As mentioned above, these elements, along with the pins on the second silicon wafer, are prevented from bonding to the glass by the metallized ground planes that extend under them, and they revert to their normal positions when the voltage is removed. Finally, the undoped regions of the second silicon wafer are dissolved in EDP, completing the batch fabrication and assembly of the micromechanisms (figure $1(f))$. Individual dice can then be snapped apart along the grooves in the glass wafer.

\section{Experimental results and discussion}

In order to illustrate the capabilities of the process, we have fabricated three separate micromotor-driven mechanisms: a coupled two-motor system, and two coupled micromotor-gear train systems (figure 2). The motors are $250 \mu \mathrm{m}, 480 \mu \mathrm{m}$ and $800 \mu \mathrm{m}$ in diameter, and the gears are $160 \mu \mathrm{m}$ and $240 \mu \mathrm{m}$ in diameter. The $800 \mu \mathrm{m}$ motors have $145 \mu \mathrm{m}$ hubs, whereas the hubs of all the other motors and gears are $110 \mu \mathrm{m}$ in diameter. The coupling links are as long as $1.2 \mathrm{~mm}$, and have pins of diameter $10 \mu \mathrm{m}$. The rotors, gears and linkage beams are all $5 \mu \mathrm{m}$ thick, and the bushings are $3 \mu \mathrm{m}$ thick. The pins on the linkages are $12 \mu \mathrm{m}$ thick, providing a $4 \mu \mathrm{m}$ clearance above the motors and gear trains. Figure 3 shows closeup optical photographs of the different mechanisms. Figure 4 shows several SEM views of these mechanisms. A number of important observations can be made about them. First, it is clear that the coupling beams are elevated above the motors and gears. The elevation and rigidity of the beams will prevent their clamping to the underlying structures from electrostatic attraction during normal operation. Second, the beams are flat in spite of

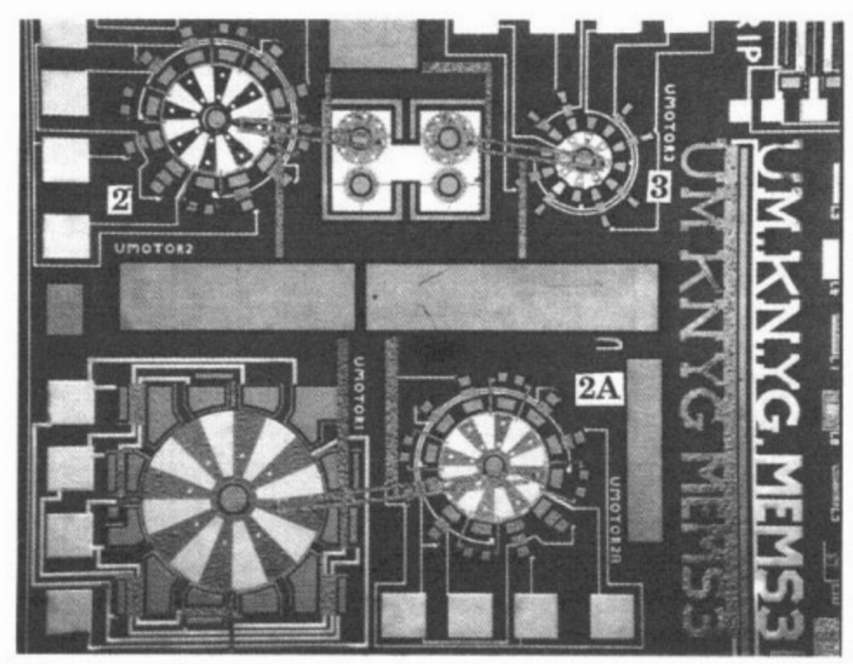

Figure 2. Photograph of micromotor-driven mechanisms: upper-half, two micromotor-gear train systems; lower-half, a coupled two-motor system. The diameters of motors 1 , 2, $2 \mathrm{~A}$ and 3 are $800 \mu \mathrm{m}, 480 \mu \mathrm{m}, 480 \mu \mathrm{m}$ and $250 \mu \mathrm{m}$, respectively. The gear diameters are $240 \mu \mathrm{m}$ and $160 \mu \mathrm{m}$. The hub diameters are $145 \mu \mathrm{m}$ for motor 1 , and $110 \mu \mathrm{m}$ for all other motors and gears. 

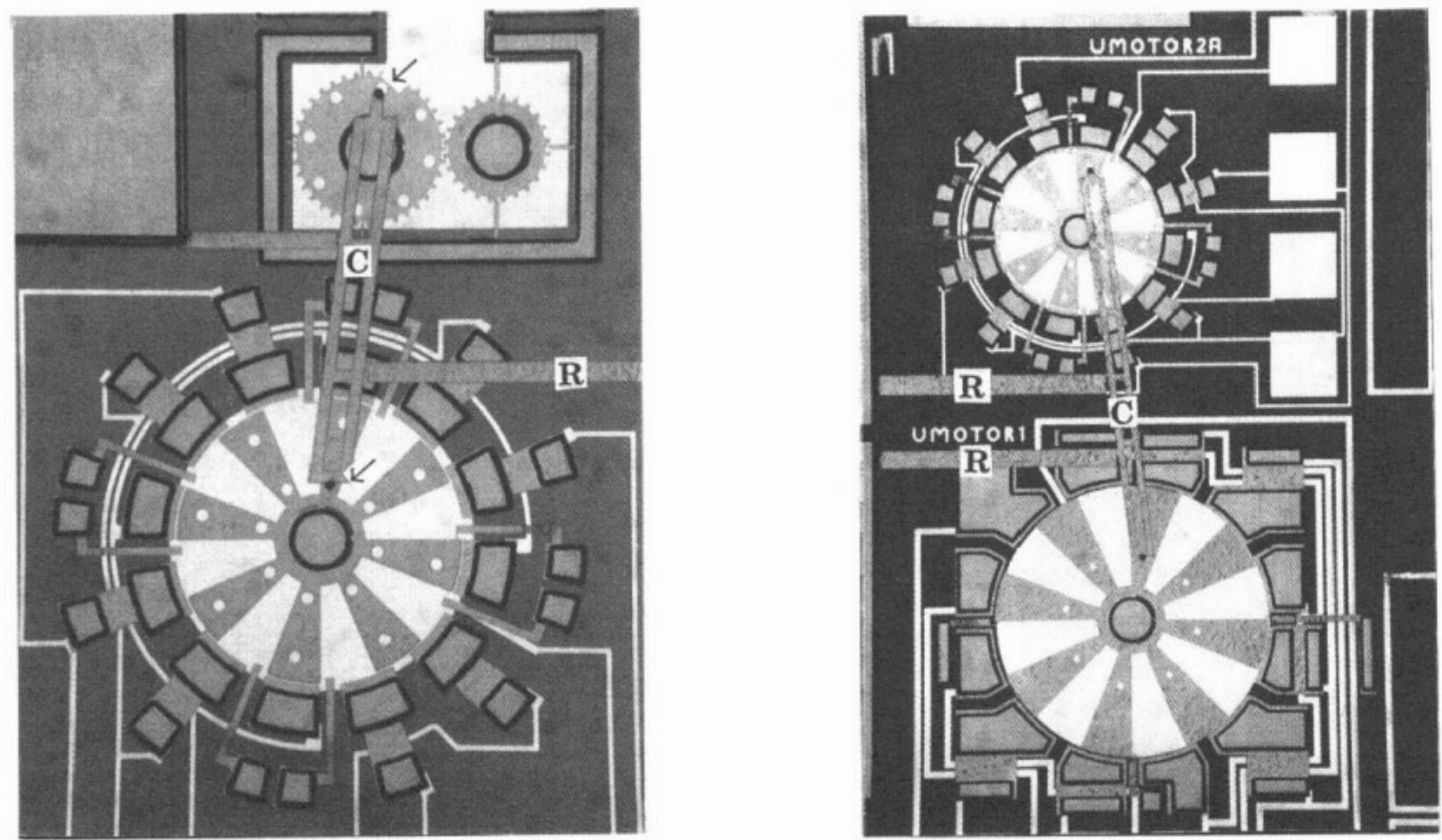

Figure 3. Close-up optical photographs of the different mechanisms shown ir figure 2. The devices are coupled by links (labelled $C$ ) that are held in place by restraints (labelled $R$ ) during processing. These restraints are destroyed by electrical or laser pulses before device operation is initiated.

their length, indicating a uniform distribution of intrinsic stress within them. Figure 5 shows a SEM view of the enmeshed teeth of two gears. As evident, the RIE has produced the nearly vertical side walls that are necessary for these elements. Although they do not address any specific mechanical application, the structures that have been fabricated demonstrate the success of the proposed technology in manufacturing thick multi-level structures incorporating selective bonding, electrical lead transfer and metal interconnect, using only five masks and ICcompatible resources.

We have successfully tested these mechanisms using direct mechanical actuation with a probe tip. As the motor is turned, the work is coupled through the linkage, causing the gears to rotate. We are at present implementing the circuitry for electrical testing of such structures. In the existing structures, of course, it is necessary to avoid dead points in the mechanism. As mentioned previously, however, with this technology it is a simple matter to design mechanisms in which the output link undergoes complete rotation.

The proposed fabrication technology has provided a significant advance towards the implementation of complete microelectromechanical systems. Although some areas remain to be refined, there are several points worth noting about the process and potential improvements that it offers.

First, the micromotors presented above do not yet include caps on top of the hubs to hold the rotors and gears in place. This is not a significant drawback since techniques for implementing these in bulk silicon technology have been presented by Suzuki [6] and Suzuki and Tanigawa [7]. A number of similar techniques are also currently under development by our group.
Second, since batch assembly of the linkages is done by alignment of the second silicon wafer with respect to the first, a minimum alignment tolerance is required between the mask that defines the structures and the perforations for the pins on the first silicon wafer (figure $1(b))$ and the mask that defines the pins on the second silicon wafer (figure 1(c)). This tolerance is determined by the aligning equipment associated with the electrostatic bonding station and by dimensional changes due to the different thermal expansion of glass and silicon when the wafers are heated during bonding. We have found that tolerances of $5 \mu \mathrm{m}$ are sufficient, but we expect to reduce these in the future.

Third, since the various elements of the linked mechanism are in relative motion, issues of friction and wear need to be further studied. Friction can be minimized if the side walls of these elements are made smooth. This is a function of the RIE mask and etch recipe [5]. As related to wear, we believe that heavily boron-doped silicon is one of the best candidates in terms of its durability and ruggedness [8].

Finally, the process presented here can very simply be extended to incorporate multiple stacked linkages at minimal extra cost $[9,10]$. Each added level requires one additional silicon wafer and two additional masks. This is an efficient and reliable method to batch fabricate and batch assemble micromechanisms, particularly those driven by micromotors.

\section{Conclusions}

We have presented a bulk silicon dissolved-wafer process for batch fabrication and assembly of micromechanisms 

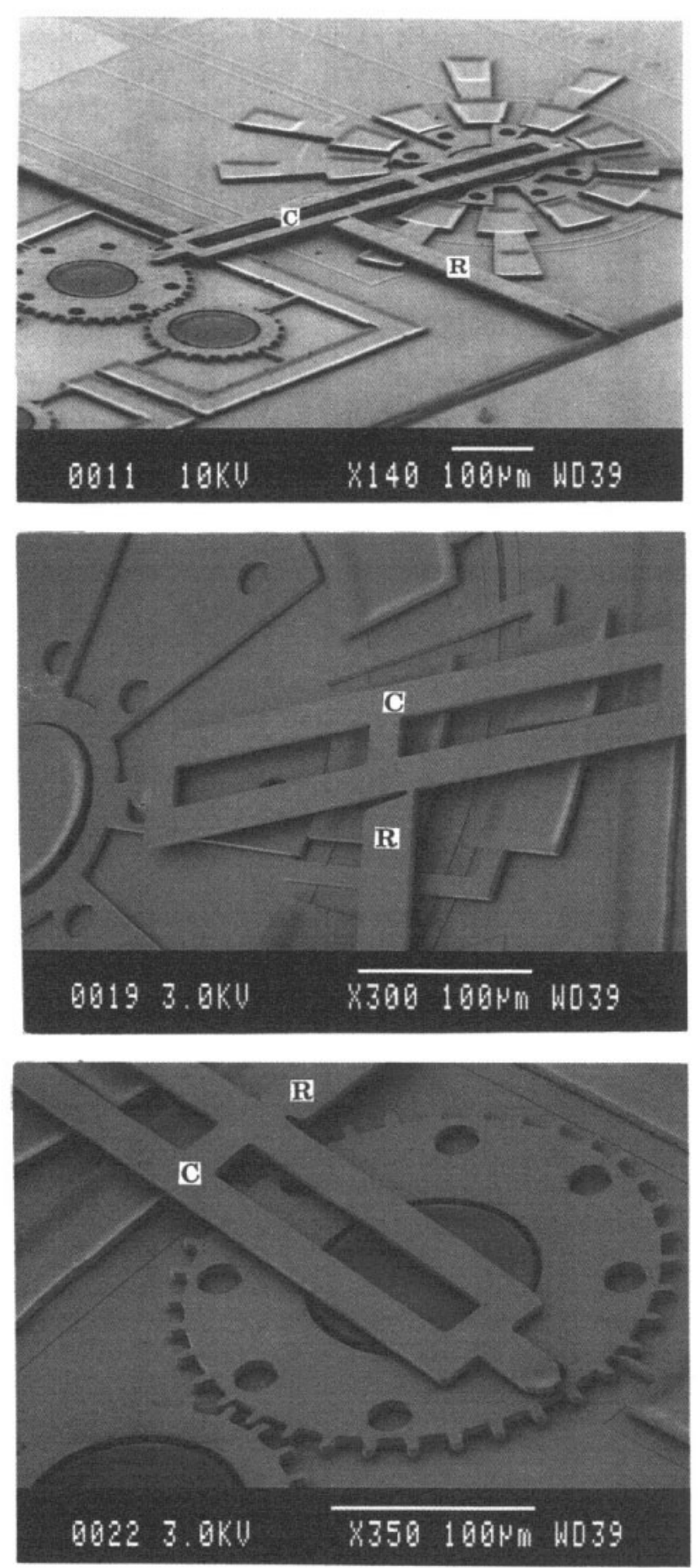

Figure 4. Oblique SEM views of mechanisms. The rotors, gears and coupling beams are all $5 \mu \mathrm{m}$ thick; the bushings and bonding recesses for the first silicon level are $3 \mu \mathrm{m}$ thick. The pins (located by the overlay arrows) on the links are $12 \mu \mathrm{m}$ thick, providing a $4 \mu \mathrm{m}$ clearance above the motors and gear trains. The restraints and the coupling beams are labelled $R$ and $C$, respectively.

with linkages. It utilizes one glass and two silicon wafers and requires five masks: one for the glass wafer and two for each silicon wafer. The first silicon wafer forms a variety of mechanical devices such as electrostatic micromotors and gear trains, while the second wafer forms links between them. The glass wafer forms the substrate

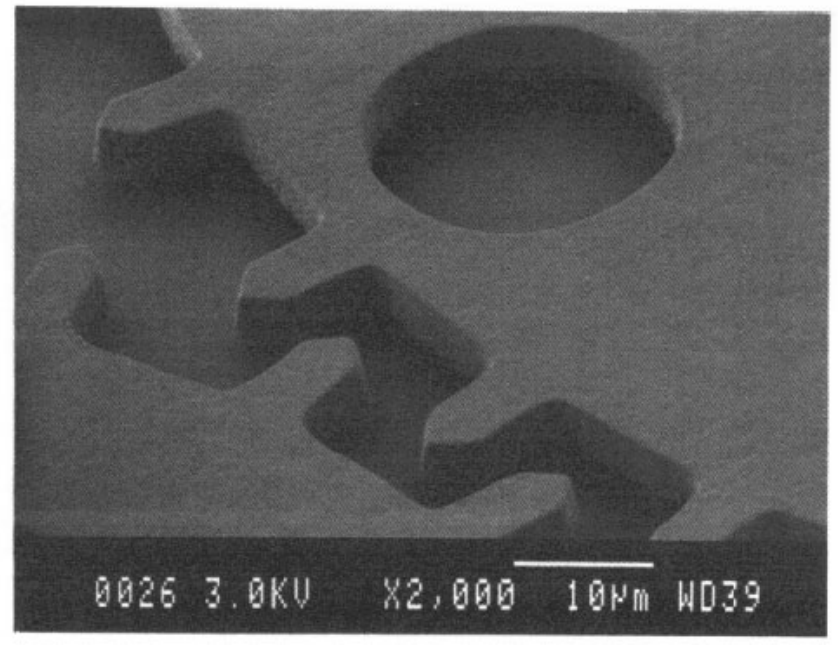

Figure 5. SEM view of the meshed teeth of two gears. As evident, the RIE has produced nearly vertical side walls.

for these mechanisms. The proposed process can be used to fabricate a variety of four-bar linkages and other mechanisms, including links that couple mechanical power out of side-driven electrostatic micromotors. Using this technology, we have batch fabricated and assembled electrostatic micromotors linked to gear trains. Tests using difect actuation with a probe tip have shown that motion of the motors is successfully transferred to the gears. We are at present implementing the circuitry required to test these structures electrically. The proposed fabrication process is well suited for mechanisms with thickness in excess of $10 \mu \mathrm{m}$.

\section{Acknowledgments}

Many helpful discussions with Professor S Kota, Dr S B Crary, Mr G K Ananthasuresh and Mr J Pittel on the overall design and requirements of the mechanisms are acknowledged. We are grateful to Professor S Pang and Mr K T Sung for suggestions regarding RIE. We also appreciate the support and encouragement of Dr George Hazelrigg of the National Science Foundation (NSF). This work was funded by NSF under grant ECS8915215.

\section{References}

[1] Mehrengany M, Phillips S M, Hsu E T and Lang J H 1991 Operation of harmonic side-drive micromotors studied through gear ratio measurements, IEEE Int. Conf. on Solid-State Sensors and Actuators (San Francisco, CA, June 1991) (New York: IEEE) Technical digest pp 59-62

[2] Guckel H, Skrobis K J, Christenson, T R, Klein J, Han S, Choi B, Lovell E G and Chapman T W 1991 Fabrication and testing of the planar magnetic micromotor J. Micromech. Microenq. 1 135-8

[3] Mabie H H and Ocvirk F W 1978 Mechanisms and Dynamics of Machinery (New York: Wiley)

[4] Hain K 1967 Applied Kinematics (New York: McGrawHill) p 23 
[5] Gianchandani Y and Najafi K 1992 Micron-sized, high aspect ratio bulk silicon micromechanical devices Proc. IEEE Workshop on Micro Electro Mechanical Systems (Travemunde, 1992) (New York: IEEE)

[6] Suzuki K 1990 Single crystal silicon micro-actuators Tech. Digest, Int. Electron Devices Meet. (New York: IEEE) (San Francisco, CA, 1990) pp 625-8

[7] Suzuki K and Tanigawa H 1991 Single crystal silicon rotational micromotors Proc. IEEE Workshop on Micro Electro Mechanical Systents (Nara, 1991) (New York: IEEE) pp 15-20

[8] Fukuda T and Ohsawa A 1991 Mechanical strength of silicon crystals with oxygen and boron impurities Appl. Phys. Lett. 58 2634-5

[9] Gianchandani Y and Najafi K 1991 A bulk silicon dissolved wafer process for microelectromechanical systems Int. Electron Devices Meet. (Washington, $D C, 1991$ ) (New York: IEEE) Technical digest

[10] Gianchandani Y and Najafi K 1992 Batch fabrication and assembly of micromotor-driven mechanisms with multi-level linkages Proc. IEEE Workshop on Micro Electro Mechanical Systems (Travemunde, 1992) (New York: IEEE) 